\title{
Transient right hemidiaphragmatic paralysis following subclavian venous catheterization: possible implications of anatomical variation of the phrenic nerve
} -a case report-

\author{
Chun Woo Yang ${ }^{1}$, Jin Sung Bae ${ }^{1}$, Tae In Park ${ }^{1}$, Jong Cheol Lee ${ }^{1}$, Jeong Eun Sohn ${ }^{1}$, Ryunga Kang ${ }^{2}$, and \\ Kye Ho Lee ${ }^{3}$
}

Department of Anesthesiology and Pain Medicine, ${ }^{1}$ Cheju Halla General Hospital, Jeju, ${ }^{2}$ Samsung Medical Center, Sungkyunkwan University School of Medicine, ${ }^{3}$ Department of Radiology, Yonsei University College of Medicine, Seoul, Korea

Phrenic nerve paralysis is an unusual complication associated with central venous catheterization. Various mechanisms have been proposed. We present a case of transient right hemidiaphragmatic paralysis after subclavian venous catheterization. We hypothesize that anatomical variation of the phrenic nerve was responsible for this complication. (Korean J Anesthesiol 2013; 65: 559-561)

Key Words: Central venous catheterization, Phrenic nerve.

Subclavian venous catheterization remains the most commonly used blind approach due to consistent landmarks, increased patient comfort, and lower potential for infection or arterial injury compared with femoral catheterization [1]. However, it may cause various complications. We present a case in which transient hemidiaphragmatic paralysis occurred after right subclavian venous catheterization and propose a mechanism that may have led to this adverse effect.

\section{Case Report}

Written informed consent and agreement for publication were obtained from the patient. A 73-year-old woman (height $153 \mathrm{~cm}$, weight $56 \mathrm{~kg}$, ASA II) was admitted to our hospital as an emergency because of acute perforation of the small bowel with hemoperitoneum. The patient's prior medical history was unremarkable. The preoperative chest x-ray was normal (Fig. 1). On emergency department admission, preoperative insertion of a central venous catheter was planned for the purpose of emergency volume resuscitation. A 7 Fr. catheter (ARROWgard Blue ${ }^{\circledR}$; Arrow International Inc., Pennsylvania, USA) was passed into the right subclavian vein through an infraclavicular approach at the first attempt. Blood returned freely through the catheter. The chest $\mathrm{x}$-ray confirmed the proper placement of the catheter,

Received: September 27, 2012. Revised: 1st, October 18, 2012; 2nd, October 25, 2012. Accepted: October 26, 2012.

Corresponding author: Jin Sung Bae, M.D., Department of Anesthesiology and Pain Medicine, Cheju Halla General Hospital, Doreongno 65, Jeju 690-766, Korea. Tel: 82-64-740-5476, Fax: 82-64-743-3110, E-mail: mdlucas@naver.com

(c) This is an open-access article distributed under the terms of the Creative Commons Attribution Non-Commercial License (http:// creativecommons.org/licenses/by-nc/3.0/), which permits unrestricted non-commercial use, distribution, and reproduction in any medium, provided the original work is properly cited. 


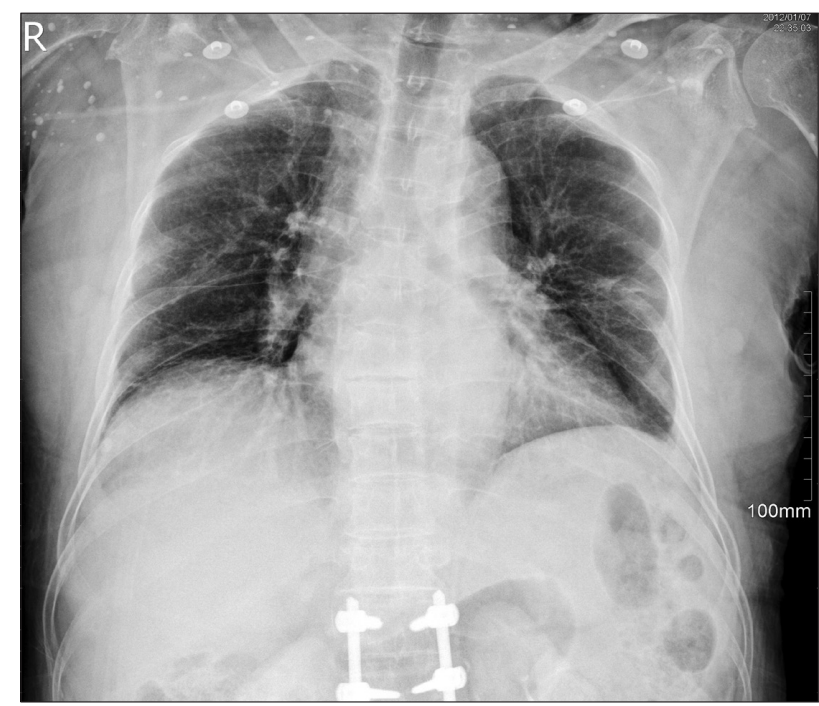

Fig. 1. The preoperative chest $\mathrm{x}$-ray.

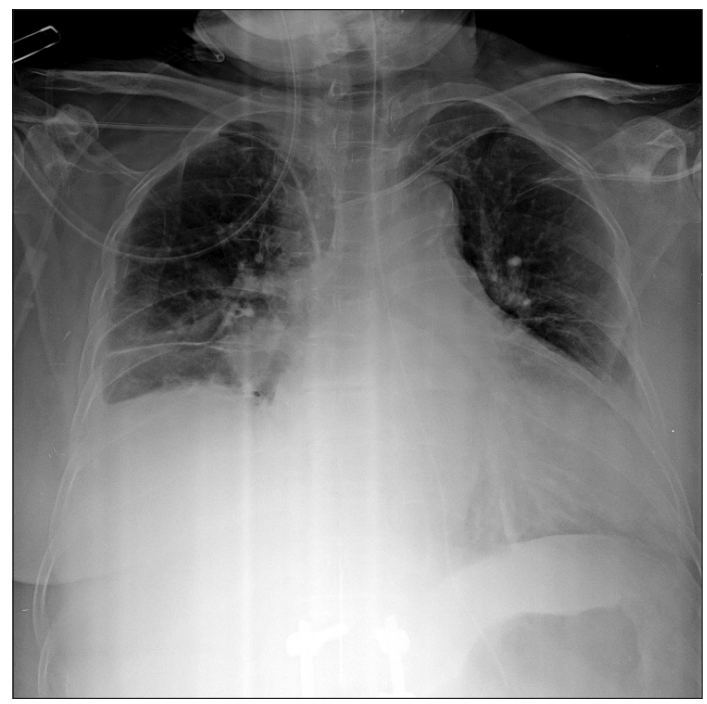

Fig. 2. Chest $\mathrm{x}$-ray on the third postoperative day showing the elevated right hemidiaphragm with pleural effusion.

but it also showed an elevation of the right hemidiaphragm. The medical team did not notice this finding. The patient underwent segmental resection of small bowel with mesenteric repair. The operation was uneventful. The patient was transferred to the intensive care unit.

On the third postoperative day, an anesthesiologist incidentally noted in a chest x-ray the elevation of the right hemidiaphragm with a small effusion (Fig. 2). The patient did not complain of any dyspnea and the arterial blood gas data were normal. Ultrasound evaluation of both diaphragms with a lowfrequency curvilinear probe as previously described [2] demonstrated a decreased motion of the right diaphragm compared

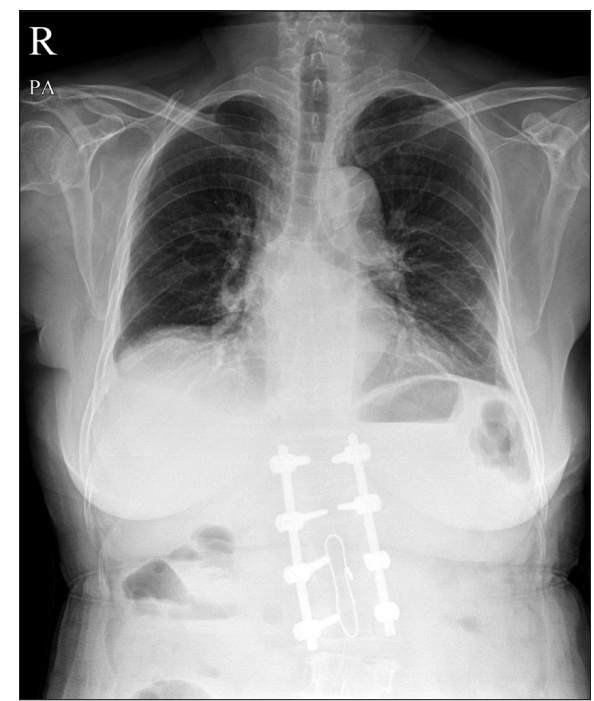

Fig. 3. Chest $x$-ray on the sixth postoperative day showing the complete restoration of the right hemidiaphragm.

with that of the left diaphragm. In $\mathrm{M}$ mode settings, left and right diaphragm excursion were $2.08 / 4.32 \mathrm{~cm}$ and $0.87 / 1.97 \mathrm{~cm}$ during quiet and deep breathing, respectively. There were no subphrenic or hepatic mass lesions.

On the fourth postoperative day, the patient was transferred to another university hospital for further treatment of the injuries. On admission, the right subclavian catheter was removed. On follow-up, a chest x-ray after the withdrawal showed the right hemidiaphragm had returned to normal (Fig. 3). Any other therapeutic intervention was not performed. The postoperative recovery was uneventful.

\section{Discussion}

Phrenic nerve paralysis following central venous catheterization is a rare complication and is not well recognized. There have been several case reports describing phrenic nerve paralysis following central venous catheterization, in which a number of mechanisms have been proposed. The mechanisms include direct nerve injury as a result of repeated attempts at venipuncture [3], compression by hematoma produced by an inadvertent arterial puncture during catheterization [4], lidocaine instillation during the procedure [5], venous wall inflammation in association with long-standing indwelling catheter [6], and rigid catheter impinging upon the thin venous wall and compressing the phrenic nerve [7].

In our case, phrenic nerve paralysis occurred immediately following right subclavian catherization and resolved completely after catheter removal. This suggests a reversible paralysis resulting from nerve compression rather than direct nerve injury to the phrenic nerve, which may cause irreversible paralysis. It is 
also unlikely that compression of the phrenic nerve by hematoma caused the hemidiaphragmatic paresis, because the central venous catheter was successfully placed at the first attempt. We hypothesize that an anatomical variation of the phrenic nerve was responsible for transient hemidiaphragmatic paralysis.

The phrenic nerve arises chiefly from the fourth cervical ventral ramus with occasional contributions from the third and fifth rami. It is formed at the upper part of the lateral border of the scalenus anterior muscle and descends almost vertically across its anterior surface behind the prevertebral fascia. At the root of the neck, it ordinarily runs anterior to the suclavian artery and posterior to the subclavian vein to enter the thorax. However, Paraskevas et al. [8] reported that the phrenic nerve crossed anterior to the subclavian vein $0.3-0.5 \mathrm{~cm}$ lateral to the jugulo-subclavian junction or traversed the anterior wall of the subclavian vein, just $1.4 \mathrm{~cm}$ lateral to the jugulo-subclavian junction. In addition, the accessory phrenic nerve may contribute to this complication. Codesido and Querri-Guttenburg [9] reported a case of accessory phrenic nerve passing through an annulus of the subclavian vein located $1 \mathrm{~cm}$ from the jugulo-subclavian junction. Similarly, Talbot [10] reported in one specimen the presence of an accessory phrenic nerve passing through the subclavian vein and dividing it into two channels. Therefore, the phrenic nerve (or accessory phrenic nerve) can be directly compressed by a rigid catheter near the jugulo-subclavian junction following catheterization, as postulated in our case. Both the successful catheterization at the first attempt and complete recovery of hemidiaphragm after catheter removal support this postulation. Paraskevas et al. [8] recommended that the puncture site should be more lateral to the outmost portion of the subclavian vein to minimize the risk of nerve impairment.

In summary, transient hemidiaphragmatic paralysis after subclavian venous catheterization can also be attributed to the anatomical variations of the phrenic nerve. Therefore, it is important to have a thorough knowledge of the relevant anatomy and anatomical variations to perform a central venous catheterization safely and efficiently.

\section{References}

1. Merrer J, De Jonghe B, Golliot F, Lefrant JY, Raffy B, Barre E, et al. Complications of femoral and subclavian venous catheterization in critically ill patients: a randomized controlled trial. JAMA 2001; 286: 700-7.

2. Gerscovich EO, Cronan M, McGahan JP, Jain K, Jones CD, McDonald C. Ultrasonographic evaluation of diaphragmatic motion. J Ultrasound Med 2001; 20: 597-604.

3. Hadeed HA, Braun TW. Paralysis of the hemidiaphragm as a complication of internal jugular cannulation: report of a case. J Oral Maxillofac Surg 1988; 46: 409-11.

4. Akata T, Noda Y, Nagata T, Noda E, Kandabashi T. Hemidiaphragmatic paralysis following subclavian vein catheterization. Acta Anaesthesiol Scand 1997; 41: 1223-5.

5. Obel IW. Transient phrenic-nerve paralysis following subclavian venipuncture. Anesthesiology 1970; 33: 369-70.

6. Rigg A, Hughes P, Lopez A, Filshie J, Cunningham D, Green M. Right phrenic nerve palsy as a complication of indwelling central venous catheters. Thorax 1997; 52: 831-3.

7. Takasaki Y, Arai T. Transient right phrenic nerve palsy associated with central venous catheterization. Br J Anaesth 2001; 87: 510-1.

8. Paraskevas GK, Raikos A, Chouliaras K, Papaziogas B. Variable anatomical relationship of phrenic nerve and subclavian vein: clinical implication for subclavian vein catheterization. Br J Anaesth 2011; 106: 348-51.

9. Codesido M, Querri-Guttenburg RA. Right accessory phrenic nerve passing through an annulus of the subclavian vein. Cin Anat 2008; 21: 779-80.

10. Talbot RW. Anatomical pitfall of subclavian venepuncture. Ann R Coll Surg Engl 1978; 60: 317-9. 